\title{
Redes sociais como meio de promoção de saúde em oncologia: um relato de experiência
}

\author{
Social networks as a means of health promotion in oncology: an experience report \\ Las redes sociales como medio de promoción de la salud em oncologia: un relato de experiencia
}

Recebido: 17/09/2021 | Revisado: 29/09/2021 | Aceito: 21/10/2021 | Publicado: 22/10/2021

\author{
Renata Cordeiro de Godoy Miranda \\ ORCID: https://orcid.org/0000-0002-7224-4173 \\ Centro Universitário Cesmac, Brasil \\ E-mail: renatagodoymiranda@ hotmail.com \\ Arthur Ravel Teles de Lima Bezerra \\ ORCID: https://orcid.org/0000-0001-7096-7899 \\ Centro Universitário Cesmac, Brasil \\ E-mail: arthurravel@ hotmail.com \\ Camila Holanda Cavalcante Matos \\ ORCID: https://orcid.org/0000-0001-8283-0590 \\ Centro Universitário Cesmac, Brasil \\ E-mail: camilaacavalcantem@outlook.com \\ Isabela Braga Peixoto \\ ORCID: https://orcid.org/0000-0003-0647-156X \\ Centro Universitário Cesmac, Brasil \\ E-mail: peixoto.isabela2@gmail.com \\ Renata Nobre da Costa \\ ORCID: https://orcid.org/0000-0002-5492-241X \\ Centro Universitário Cesmac, Brasil \\ E-mail: renata_nobree@hotmail.com \\ Beatriz Cordeiro de Godoy Miranda \\ ORCID: https://orcid.org/0000-0001-9175-7153 \\ Centro Universitário Cesmac, Brasil \\ E-mail: beaatrizgodoy@gmail.com \\ Isabela Vieira Melo \\ ORCID: https://orcid.org/0000-0001-8440-1386 \\ Centro Universitário Cesmac, Brasil \\ E-mail: isabelavieiramelo@hotmail.com \\ Vitória Lívia Marinho de Oliveira \\ ORCID: https://orcid.org/0000-0002-4935-3339 \\ Centro Universitário Cesmac, Brasil \\ E-mail: vit.livia@hotmail.com \\ Camila Maria Beder Ribeiro Girish Panjwani \\ ORCID: https://orcid.org/0000-0003-0203-3079 \\ Centro Universitário Cesmac, Brasil \\ E-mail: camila.panjwani@cesmac.edu.br \\ Fernanda Braga Peixoto \\ ORCID: https://orcid.org/0000-0003-4549-4959 \\ Centro Universitário Cesmac, Brasil \\ E-mail: fernanda.peixoto@cesmac.edu.br
}

\section{Resumo}

As redes sociais têm crescido exponencialmente nas últimas décadas como meio de trabalho e, com eles, o uso de aplicativos para a promoção de saúde. Apesar do modelo de atenção à saúde atual ainda ser centrado na assistência curativa individual, nota-se a importância de haver uma mudança tornando-o preventivo, uma vez que esse, sozinho, não tem resolvido os problemas de saúde da população. O câncer causa preocupação nos profissionais de saúde por ser uma doença que tem um avanço silencioso e os indivíduos são diagnosticados em estado avançado, demonstrando importância de haver a estimulação de cuidados profiláticos. As mídias em detrimento dos avanços tecnológicos podem ser utilizadas como estratégias de promoção a saúde em oncologia uma vez que possibilitam a globalização das informações geradas em tempo real. Dessarte, permite que pessoas com contextos socioculturais diferentes consigam interagir, contribuindo para que o processo de aprendizagem se torne mais eficaz, fidedigno e menos maçante, proporcionando assim uma melhor elaboração sobre as estratégias de enfrentamento e solução de problemas que necessitam ser superados pela sociedade. O projeto teve como objetivo mobilizar o público geral, incluindo estudantes e colaboradores do Centro Universitário Cesmac, para buscarem uma relação mais consciente sobre o câncer. Foram realizadas aulas e atividades de educação em saúde abrangendo as áreas da odontologia, medicina, 
nutrição, psicologia e enfermagem. As ações foram realizadas por meio de aulas na plataforma digital Microsoft teams com convidados diversos, grupo de discussão de manejo terapêutico multidisciplinar e postagens informativas na plataforma digital instagram.

Palavras-chave: Redes sociais; Promoção de saúde; Oncologia.

\begin{abstract}
Social networks have grown exponentially in recent decades as a means of work and, with them, the use of applications for health promotion. Although the current health care model is still centered on individual curative care, the importance of changing to preventive care is noted, as this alone has not solved the population's health problems. Cancer is a concern among health professionals because it is a disease that has a silent advance and individuals are diagnosed in an advanced state, demonstrating the importance of stimulating prophylactic care. The media, to the detriment of technological advances, can be used as health promotion strategies in oncology as they enable the globalization of information generated in real time. Thus, it allows people with different sociocultural contexts to interact, contributing to the learning process becoming more effective, reliable and less boring, thus providing a better elaboration on coping strategies and problem solving that need to be overcome by society. The project aimed to mobilize the general public, including students and employees of the Cesmac University Center, to seek a more aware relationship about cancer. Health education classes and activities were held covering the areas of dentistry, medicine, nutrition, psychology and nursing. The actions were carried out through classes on the Microsoft teams digital platform with several guests, a multidisciplinary therapeutic management discussion group and informative posts on the Instagram digital platform.
\end{abstract}

Keywords: Social networks; Health promotion; Oncology.

\title{
Resumen
}

Las redes sociales han crecido exponencialmente en las últimas décadas como medio de trabajo y, con ellas, el uso de aplicaciones para la promoción de la salud. Si bien el modelo actual de atención a la salud aún se centra en la atención curativa individual, se destaca la importancia de pasar a la atención preventiva, ya que ésta por sí sola no ha resuelto los problemas de salud de la población. El cáncer es una preocupación entre los profesionales de la salud ya que es una enfermedad que tiene un avance silencioso y los individuos son diagnosticados en un estado avanzado, lo que demuestra la importancia de estimular la atención profiláctica. Los medios de comunicación, en detrimento de los avances tecnológicos, pueden utilizarse como estrategias de promoción de la salud en oncología ya que permiten la globalización de la información generada en tiempo real. Así, permite interactuar a personas con diferentes contextos socioculturales, contribuyendo a que el proceso de aprendizaje sea más efectivo, confiable y menos aburrido, brindando así una mejor elaboración de estrategias de afrontamiento y resolución de problemas que deben ser superados por la sociedad. El proyecto tenía como objetivo movilizar al público en general, incluidos estudiantes y empleados del Centro Universitario Cesmac, para buscar una relación más consciente sobre el cáncer. Se impartieron clases y actividades de educación sanitaria en las áreas de odontología, medicina, nutrición, psicología y enfermería. Las acciones se realizaron a través de clases en la plataforma digital de equipos de Microsoft con varios invitados, un grupo de discusión de gestión terapéutica multidisciplinar y publicaciones informativas en la plataforma digital de Instagram.

Palabras clave: Redes sociales; Promoción de la salud; Oncología.

\section{Introduçãa}

Há algum tempo o câncer causa preocupação nos profissionais de saúde por ser uma doença que, muitas vezes, tem avanço silencioso e os indivíduos geralmente são diagnosticados em estado avançado, sendo necessário haver a estimulação de cuidados profiláticos. Para o Brasil, a estimativa aponta que ocorrerão 625 mil novos casos de câncer por cada ano do triênio 2020-2022. A maior incidência será do câncer de pele não melanoma, seguido pelos cânceres de mama e próstata (INCA, 2020).

A Organização Mundial da Saúde (OMS) define três diferentes níveis de atenção à saúde: primário, secundário e terciário, cada um foca no atendimento ao paciente de acordo com sua necessidade, tal modelo de organização é seguido pelo Brasil (Soldá, 2018). No caso dos pacientes oncológicos, devido aos exames mais invasivos, procedimentos de alto custo e tecnologia, fazem parte da atenção terciária ou Alta Complexidade. Nesse contexto, é notório que o modelo de atenção à saúde atual ainda é centrado na assistência curativa individual, assim, nota-se a importância de haver uma mudança tornando-o preventivo e abordá-lo no nível primário, uma vez que esse, sozinho, não tem resolvido os problemas de saúde da população. 
A saúde é produzida por meio das interações sociais, dessa forma, em detrimento dos avanços tecnológicos, as redes sociais podem ser utilizadas como estratégias de promoção a saúde em oncologia uma vez que possibilitam a globalização das informações geradas em tempo real. Neste sentido, é importante entender em que medida o engajamento de indivíduos com doenças crônicas em ambientes virtuais pode influenciar positivamente aos cuidados profiláticos, uma vez que é uma das problemáticas dos pacientes oncológicos que só descobrem a doença em sua forma mais avançada (Fernandes, 2018).

Um fator essencial que deve ser avaliado nesses meios de comunicação é a qualidade, profundidade e um fator humanizado das informações compartilhadas, de modo que aproxime e passe segurança, como também demonstrar conhecimento e autoridade sobre o assunto (Toquarto, 2002). Além de tudo, a pandemia do novo coronavírus afastou as pessoas do contato pessoal, mas como todos os outros problemas de saúde pública, a atenção ao paciente oncológico não pode ser afastada, nesses casos ela tem que estar cada vez mais presente, consequentemente, a internet faz com que as pessoas possam se conectar mesmo estando distantes, até quando o assunto é saúde, que também pode ser feita por meio virtual, sem substituir o modo presencial, mas como uma alternativa para se reinventar o cuidado (Brito, 2020).

Desse modo, o presente estudo justifica-se pela importância de relatar o modelo de promoção de saúde nas redes sociais, a qual alcança várias pessoas de diferentes classes sociais, com o intuito de abordar a temática voltada não apenas a assistência curativa, mas também para prevenção. Sendo assim, tem como objetivo compartilhar a experiência da fundação e das atividades de uma liga acadêmica pelo Centro Universitário Cesmac, a qual aborda, de forma multidisciplinar, sobre o câncer e os pacientes oncológicos nas redes sociais.

\section{Metodologia}

Para Pereira et al. (2018) um trabalho científico visa trazer algum saber novo e, no presente estudo procura-se trazer um relato de experiência, caracterizado como um estudo descritivo, de abordagem qualitativa.

Relata-se a realização e a excussão de uma extensão universitária, de forma remota, durante o período de isolamento social ocasionado pela Pandemia do Coronavírus. Formada por acadêmicos e discentes do curso de Enfermagem, Medicina, Nutrição, Odontologia e Psicologia, de uma Instituição de Nível Superior do Estado de Alagoas. Foi criado um perfil em uma rede social e postado diversas temáticas relacionadas à oncologia.

\section{Relato de Experiência}

A liga acadêmica interdisciplinar de oncologia (LAION) é constituída por alunos de Enfermagem, Odontologia, Psicologia, Nutrição e Medicina de diferentes períodos do Centro universitário Cesmac no estado de Alagoas. Foi fundada em julho de 2020 com o objetivo de discutir e aprofundar o conhecimento sobre o câncer e seu tratamento de forma integrada através do tripé acadêmico de ensino, pesquisa e extensão sob orientação e supervisão de profissionais especialistas e de maneira remota, uma vez que o cenário atual é pandêmico. A motivação que levou a criação da liga foi, além da disseminação de conhecimento para a população, fidelizar e esclarecer a importância de um tratamento interprofissional para a recuperação do paciente oncológico e a melhoria do seu prognóstico.

Assim, definiu-se um cronograma de aulas para serem ministradas pela plataforma digital Microsoft Teams (Figura 1) e assuntos a serem debatidos, intercalando uma aula ministradas por um profissional da área, onde após a mesma ocorre um momento em que os alunos tiram as suas dúvidas com o palestrante (Figura 2), e uma aula ministrada por um aluno de cada curso para discutir o tratamento o tema abordado na semana anterior. 
Figura 1: Sala do Microsoft teams.

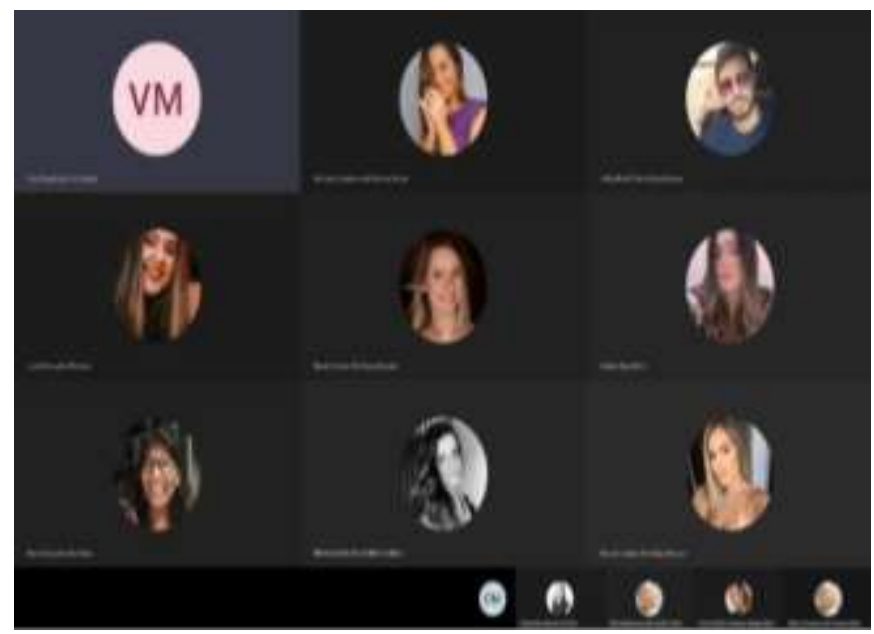

Fonte: Autores.

Figura 2: Convite para a aula do profissional da área.

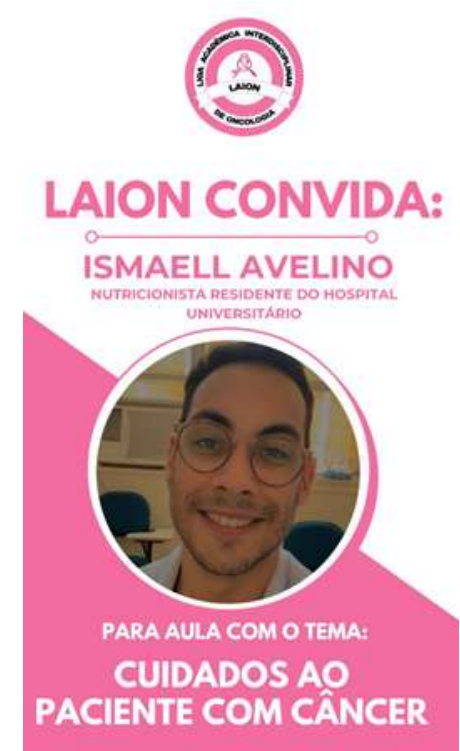

Fonte: Autores.

Os encontros são semanais e no fechamento do assunto dois membros da liga ficam responsáveis por realizar um resumo detalhado sobre o que foi abordado e disponibilizar para todo o grupo através de um Google Drive (Figura 3). O material do resumo é transformado em sequências de posts para Instagram (figura 4 e 5) a fim de desmistificar o assunto, que ainda é um tabu, para a sociedade de forma geral. São liberados de um a dois posts por semana e cada semana é um tema diferente. 
Figura 3: Pasta no google drive.

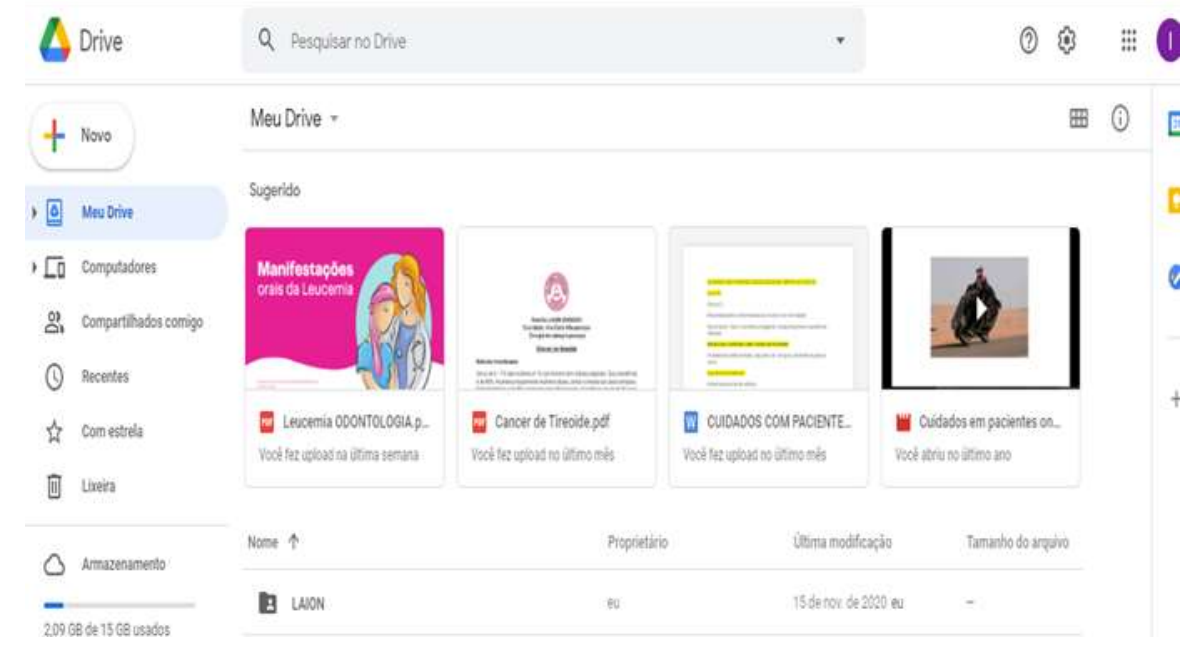

Fonte: Autores.

Figura 4: Post explicativo sobre câncer de tireóide.

Figura 5: Feed do Instagram da liga.

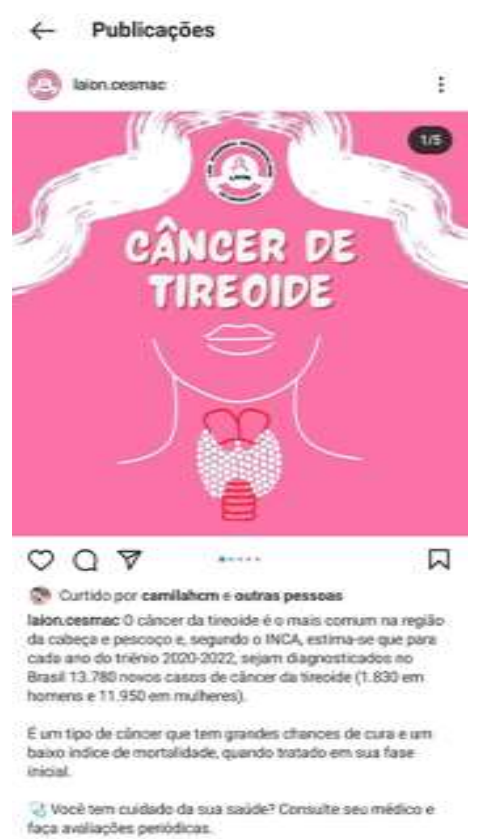

Fonte: Autores.

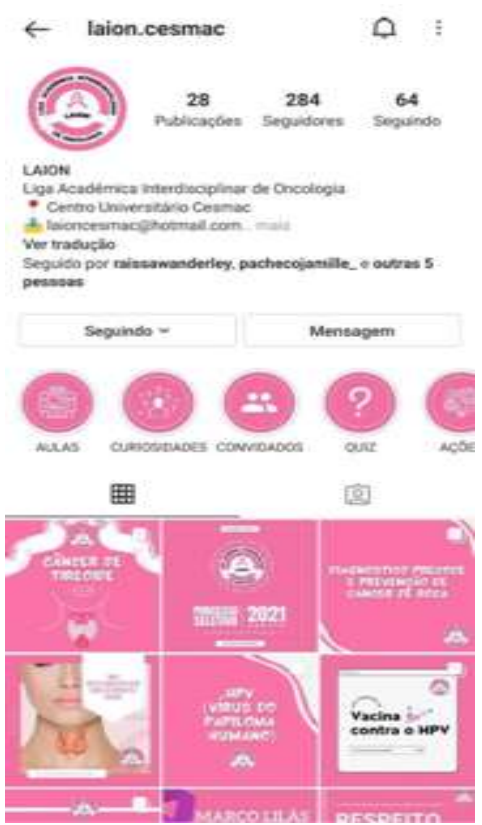

Fonte: Autores.

Iniciaram-se os estudos a partir da oncogênese, formação tumoral, e deu continuidade seguindo os principais tipos de câncer definidos pelo INCA-Instituto Nacional de Câncer, sendo eles câncer de mama e próstata. Além disso, foi levado a debate o morrer simbólico após o diagnóstico, os cuidados paliativos, o cuidado com o paciente oncológico em tempos de Covid-19 e os cuidados e prevenção contra o papilomavírus humano (HPV).

Os palestrantes são escolhidos através de suas especialidades e qualificações, garantindo assim uma abordagem segura e completa para os alunos. Esses usam de material em Power Point e liberam referências para que os alunos possam aprofundar seus estudos. Além disso, todas as aulas ficam gravada na plataforma Microsoft Teams mediante a autorização prévia do profissional. 
Com a facilidade de comunicação que o meio online nos permite, a LAION vem sendo disseminada pelo Brasil todo e conta com profissionais de diferentes lugares trazendo suas vivências, ampliando mais ainda o horizonte de conhecimento.

\section{Discussão}

A Extensão Universitária é um método educativo, cultural e científico, o qual integra o ensino e a pesquisa de maneira intrínseca proporcionando um encadeamento inovador entre Universidade e sociedade. Esse vínculo beneficia o processo educacional socializando o conhecimento acadêmico com a participação da comunidade na vida acadêmica (Nogueira, 2013; Carneiro, 2015). Os discentes, sob a orientação de docentes, firmam-se na obtenção de uma visão mais crítica e ampliada da profissão, concebendo novas abordagens e possibilidades na atenção à saúde (Carneiro, 2015).

Com o início da pandemia de COVID-19, profissionais de diferentes áreas sentiram a necessidade de ressignificar o uso de ferramentas já conhecidas anteriormente, como as redes sociais, serviços de streaming e recursos de plataformas como Google e Microsoft (Aquino, 2020). Sabe-se que um dos campos afetados foi o de educação, uma vez que as atividades, anteriormente presenciais, passaram a ter necessidade de reajustes (Dias, 2021).

Além de tudo, a pandemia do novo coronavírus afastou as pessoas do contato pessoal, mas como todos os outros problemas de saúde pública, a atenção ao paciente oncológico não pode ser afastada, nesses casos ela tem que estar cada vez mais presente, consequentemente, a internet faz com que as pessoas possam se conectar mesmo estando distantes, até quando o assunto é saúde, que também pode ser feita por meio virtual, sem substituir o modo presencial, mas como uma alternativa para se reinventar o cuidado (Brito, 2020).

A saúde é produzida por meio das interações sociais, dessa forma, em detrimento dos avanços tecnológicos, as redes sociais podem ser utilizadas como estratégias de promoção a saúde em oncologia uma vez que possibilitam a globalização das informações geradas em tempo real. Neste sentido, é importante entender em que medida o engajamento de indivíduos com doenças crônicas em ambientes virtuais pode influenciar positivamente aos cuidados profiláticos, uma vez que é uma das problemáticas dos pacientes oncológicos que só descobrem a doença em sua forma mais avançada (Fernandes, 2018).

Foi registrado pelos responsáveis do Instagram que a plataforma é a quinta rede social mais usada a nível global, ficando atrás do Facebook, do Youtube, do Whatsapp e do WeChat. Além disso, numa pesquisa feita pela companhia Statista, demonstrou-se os países em que possuíam mais usuários conectados no aplicativo Instagram no primeiro mês de 2021, o Brasil ocupou o terceiro lugar no ranking das nações (ABC repórter, 2021).

Seguindo esse raciocínio, a promoção de saúde em mídias virtuais é uma das áreas que tem crescido exponencialmente no último ano no país. Profissionais e acadêmicos de saúde utilizam suas contas pessoais e/ou profissionais para levar informações acerca de diferentes temas, de uma maneira simplificada e utilizando linguagem de fácil entendimento, com o intuito de abranger um público-alvo maior (Silva, 2020).

Assim, os membros da Liga Acadêmica Interdisciplinar de Oncologia do Centro Universitário Cesmac, frente a essa perspectiva, trouxeram diferentes formas de promoção de saúde em oncologia desmistificando temas voltados para a oncologia, viabilizando não apenas aumentar a atenção à prevenção de diferentes tipos de câncer, como também a procura de profissionais de saúde para uma melhora de prognóstico em casos de câncer pré-existentes em estado não avançado.

\section{Conclusão}

Diante do exposto, fica claro a necessidade da existência de um meio de comunicação mais acessível para a promoção de conhecimento, que possa ser disseminado de modo mais didático e através de uma fonte confiável, visto que a informação, através das redes sociais, se expande e se perpetua de forma universal, alcançando assim uma variedade maior de pessoas. Fica claro que a proposta de levar saúde de foma digital atinge o objetivo de prevenção e o estímulo ao autocuidado. 
Visto que as redes servem, além de local para buscar conhecimento, como um espaço onde os pacientes oncológicos possam compartilhar suas experiencias e buscar acolhimento, é sugerido o aprimoramento de espaços onlines específicos para esse população e maiores estudos do impacto das redes sociais como forma de promoção de saúde.

\section{Referências}

ABC Reporter. (2021). Brasil é o $3^{\circ}$ país com mais usuários no Instagram em 2021. Disponível em: https://abcreporter.com.br/2021/04/15/brasil-e-o-3o-paiscom-mais-usuarios-ativos-no-instagram-em-2021/

Aquino, E. M., Silveira, I. H., Pescarini, J. M., Aquino, R., Souza-Filho, J. A. D., Rocha, A. D. S. \& Lima, R. T. D. R. S. (2020). Medidas de distanciamento social no controle da pandemia de COVID-19: potenciais impactos e desafios no Brasil. Ciência \& Saúde Coletiva, $25,2423-2446$.

Bayde, L., Freitas, R., Costa, A. \& Felix, M. (2020). Tecnologia e mídias como saídas em uma pandemia: um foco em possibilidades multidisciplinares e interdisciplinares. Revista Sistemas e Mídias Digitais, 5(1).

Bispo, D. B., Junior, E. J. P., Gonçalves, H. C. B., da Silva Lourenço, J., Migliavacca, I. S., Teixeira, M. L. S. \& Fernandes, L. C. (2020). Utilização das mídias digitais para ensino, pesquisa e extensão: atuação das ligas acadêmicas no contexto da pandemia. Mostra científica de ações extensionistas, 5(1), 96101.

Brito, B. P. M., Pacheco, E. M., \& da Silveira, L. (2020). Rede Convida: estendendo zelo e outros elos em tempos de pandemia. (Des) troços: revista de pensamento radical, 1(1), 24-41.

Carneiro, J. A., da Costa, F. M., Poswar, F. D. O. \& de Freitas, M. O. S. (2014). Liga acadêmica: instrumento de ensino, pesquisa e extensão universitária. Gestão e Saúde, 6(1), 667.

Silva, M. M. S., de Carvalho, K. G., da Silva Cavalcante, I. K., Saraiva, M. J. G., da Conceição Lomeo, R. \&Vasconcelos, P. R. (2020). Interseção de saberes em mídias sociais para educação em saúde na pandemia de Covid-19. SANARE-Revista de Políticas Públicas, 19(2).

Melo, M. C., da Fonseca, C. M. F. \& Vasconcellos-Silva, P. R. (2017). Internet e mídias sociais na educação em saúde: o cenário oncológico. Cadernos do Tempo Presente, 27(1), 69-83.

Dias, É. (2021). A Educação, a pandemia e a sociedade do cansaço. Ensaio: avaliação e políticas públicas em educação, 29(112).

Fernandes, L.S., Calado, C. \& Araújo, A.S. (2018). Redes sociais e práticas em saúde: influência de uma comunidade online de diabetes na adesão ao tratamento. Ciência e Saúde Coletiva, 23(10)

INCA. (2020). Estimativa 2020. Disponível em: https://www.inca.gov.br/estimativa/introducao.

Melo, J. M. F. O., Bione, G. B. B.S., Almeida, F. R., Pereira, F. Q., Silveira, R. C. J. \& Vajgel, B. C. F. (2020). Consulta remota na odontologia: como a telessaúde pode diminuir os desafios enfrentados pela covid-19? Uma revisão de literatura. Odontologia Clínico-Científica, 19(3), $262-267$.

Nogueira, M. D. D. P. (2013). O Fórum de Pró-reitores de Extensão das Universidades Públicas Brasileiras: um ator social em construção. Interfaces-Revista de Extensão da UFMG, 1(1), 35-47.

Pereira, A.S. et al. (2018). Metodologia da pesquisa científica. [e-book]. Santa Maria. Ed. UAB/NTE/UFSM. https://repositorio.ufsm.br/bitstream/handle/1/15824/Lic_Computacao_MetodologiaPesquisa-Cientifica.pdf?sequence=1.

Ramos, R.S. (2020). A enfermagem oncológica no enfrentamento da pandemia de covid-19: reflexões e recomendações para a prática de cuidados em oncologia. Revista Brasileira de Cancerologia, 66(1).

Rocha, L. C. F., Riquelme, C. S. G., Pessanha, L. C., Souza, K. C. B. A. \& Assunção, L. R. (2021). O papel das ligas acadêmicas durante a pandemia de covid-19 e aulas online. Ciências biológicas da saúde, 130-133.

Silva, T. S., Matos, C. H. C., Costa, M. O. S., Miranda, R. C. G., Bezerra, A. R. T. L, Santos, C. M. L., Fernandes, G. M. C. B. S., Neto, J. D. S., Silva, M. C. N., Sarmento, M. E. L. M., Amaral, A. L. C. \& Monteiro, N. R. (2021). Relato de experiência do projeto de extensão universitária remota: readaptações - um olhar resiliente em meio a pandemia. Research, Society and Development, 10(8).

Silva, A. F., Estrela, F. M., Lima, N. S. \& Abreu, C. T. A. (2020). Saúde mental de docentes universitários em tempos de pandemia. Physis: Revista de Saúde Coletiva, 30(02).

Soldá, L. (2018). Conheça os níveis de atenção à saúde e estratégias de gestão. https://proxis.com.br/conheca-os-niveis-de-atencao-a-saude-e-estrategias-degestao/.

Torquato, G. (2002). Tratado de comunicação organizacional e política. São Paulo: Pioneira.

Vermelho, S. C., Velho, A. P. M., Bonkovoski, A. \& Pirola, A. (2014). Refletindo sobre as redes sociais digitais. Educação \& Sociedade, 35(1), 179-196. 